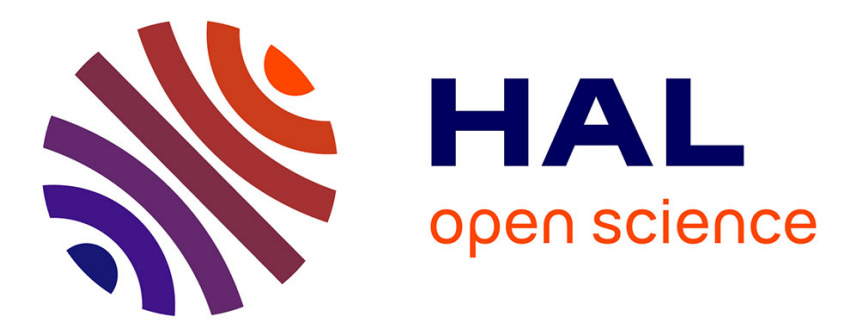

\title{
Paternité tardive: un risque en matière de reproduction?
}

Patrick Thonneau, Elise de La Rochebrochard

\section{To cite this version:}

Patrick Thonneau, Elise de La Rochebrochard. Paternité tardive: un risque en matière de reproduction?. Basic and clinical andrology, 2007, 17 (2), pp.117-122. 10.1007/BF03041164\#rightslink . hal-02265218

\section{HAL Id: hal-02265218 \\ https://hal.science/hal-02265218}

Submitted on 8 Aug 2019

HAL is a multi-disciplinary open access archive for the deposit and dissemination of scientific research documents, whether they are published or not. The documents may come from teaching and research institutions in France or abroad, or from public or private research centers.
L'archive ouverte pluridisciplinaire HAL, est destinée au dépôt et à la diffusion de documents scientifiques de niveau recherche, publiés ou non, émanant des établissements d'enseignement et de recherche français ou étrangers, des laboratoires publics ou privés. 


\title{
Paternité tardive : un risque en matière de reproduction ?
}

\author{
Patrick THONNEAU1, Elise de LA ROCHEBROCHARD ${ }^{2}$
}

\author{
1 Equipe Accueil N³6 94 " Recherche en Fertilité Humaine ", Université Paul Sabatier - \\ Toulouse III / CHU Purpan Toulouse \\ 2 INED-INSERM Nº22, Le Kremlin-Bicêtre
}

\section{RÉSUMÉ}

Parallèlement au risque reproductif bien documenté lié à l'âge maternel, des publications croissantes font état d'une majoration significative d'issues reproductives défavorables lié à l'âge paternel. Ce travail de synthèse fait une analyse des principaux travaux scientifiques récents sur le sujet, attestant d'une implication réelle de l'âge paternel au delà de $\mathbf{4 0}$ ans sur la reproduction humaine.

Mots clés : fertilité, fertilité masculine, reproduction, âge paternel, épidémiologie

\section{INTRODUCTION}

Si les effets de l'âge maternel, en particulier après 35 ans, sur la reproduction ont été largement démontrés (délai de conception, fausse-couche, grossesse extrautérine, malformation), il en va tout autrement pour l'âge paternel. D'un côté, très peu de publications scientifiques concernant les aspects masculins, de l'autre des images de stars masculines pères à un âge avancé - l'influence de l'âge sur la reproduction ne semble donc a priori toucher que la composante féminine de l'espèce humaine !

Toutefois, plusieurs articles récents et une analyse détaillée de la littérature existante permettent d'apporter un éclairage nouveau sur l'implication de l'âge paternel sur la reproduction humaine. Quatre indicateurs clés de la reproduction ont été analysés : les caractéristiques du sperme, l'infécondité (mesurée par le délai de conception et par le taux de grossesses cliniques en assistance médicale à la procréation (AMP)), le taux de fausses-couches et le taux de malformations congénitales.

\section{AGE PATERNEL ET MODIFICATIONS DU SPERME}

Dans ce domaine, peu de travaux ont été menés avec essentiellement des études cliniques (les comparaisons portant entre des hommes jeunes $<30$ ans et plus âgés > 50 ans), sans notion de seuil critique et avec une grande variabilité dans les résultats. Les deux principales publications (dont l'une est une revue de la littérature) concluent à une diminution du volume spermatique (de 3 à $22 \%$ ), de la mobilité (de 3 à $37 \%$ ) et du pourcentage de formes normales (de 4 à 18\%) $[3,16]$.

\section{AGE PATERNEL ET INFÉCONDITÉ}

En matière d'infécondité, plusieurs indicateurs peuvent être utilisés. Les publications sur l'âge paternel portent sur les deux indicateurs majeurs de l'infécondité : d'une part, le délai de conception (Time To Pregnancy, $T T P$ ), exprimé en nombre de mois écoulés entre le début de l'exposition à la grossesse et le début de celle-ci, et d'autre part le taux de grossesses dans le cadre des AMP.

\section{Augmentation du délai de conception en fonction de l'âge paternel \\ Une publication assez ancienne (1990), menée au}

\section{Correspondance :}

Dr Patrick THONNEAU - Equipe Accueil N 3694 , Hôpital Paule de Viguier, TSA 70034, 31059 Toulouse Cedex 9 Email thonneau.p@chu-toulouse.fr 
Danemark auprès de 8338 couples et utilisant comme indicateur un délai de conception supérieur à 12 mois ne montrait pas d'effet significatif de l'âge paternel [19]. Cette publication présentait cependant le biais de porter sur des naissances vivantes ce qui pourrait expliquer l'absence d'effet de l'âge paternel sur le délai de conception. En 1994, une équipe australienne a trouvé, en utilisant un délai de conception de 9 mois, un risque de $2,3(\mathrm{OR}=2,3 ; 1,4-3,7)$ chez les hommes âgés de plus de 35 ans comparés à ceux ayant moins de 35 ans [10].

En 2000, une équipe anglaise a montré que la probabilité de conception était divisée par 2 chez les hommes âgés de 40 ans comparés à ceux âgés de 24 ans [11]. Plus récemment, une équipe française a analysé le délai de conception chez 3287 femmes européennes âgées de 25 à 44 ans, en utilisant comme classe de référence la classe d'âge 20-29 ans. Les auteurs montrent que le risque de conception au delà de 12 mois est multiplié par $2,9(\mathrm{OR}=2,9 ; 1,8-4,9)$ lorsque l'homme est âgé de 40 ans et plus, et sa compagne ayant un âge compris entre 35 et 39 ans [7].

\section{Taux de grossesses cliniques en AMP en fonction de l'âge paternel}

Une première publication d'une équipe française, en 1990, montrait une diminution du taux de grossesses cliniques avec l'âge paternel [26]. Toutefois, à la même époque, deux études internationales ne retrouvaient pas d'effet paternel sur le taux de grossesses cliniques $[12,20]$.

Une étude récente (2006) menée à partir de la base FIVNAT française a analysé le risque d'échec de conception en fonction de l'âge de la femme, de l'homme puis en tenant compte de l'interaction entre l'âge maternel et paternel [4]. Cette étude a inclus 1938 hommes, dont la partenaire était totalement stérile (absence des deux trompes ou occlusion tubaire bilatérale) et inscrits dans un programme de FIV. Comme illustré dans le Tableau 1, on retrouve une majoration du risque de ne pas concevoir chez la femme à partir de 38 ans et au delà de 40 ans chez l'homme. En prenant en compte l'interaction âge maternel et paternel, les résultats font état d'une multiplication du risque de ne pas concevoir de 5,7 lorsque la femme et l'homme sont âgés de plus de 40 ans.

\section{AGE PATERNEL ET FAUSSES-COUCHES}

Plusieurs publications internationales récentes ont mis en avant une augmentation du risque de faussescouches avec l'âge paternel.

Une étude australienne réalisée en 1994 et portant sur
484 grossesses, a trouvé un risque multiplié par 2,3 chez les hommes de plus de 35 ans comparés à ceux ayant moins de 35 ans [10]. En 2003, une étude française incluant 2414 grossesses a retrouvé également une majoration du risque de fausses-couches chez les hommes de plus de 35 ans $(O R=2,1)[25]$.

Enfin, une large étude européenne incluant 3174 grossesses a montré une multiplication du risque par $6,7(3,5-12,9)$ chez des couples où l'homme avait 40 ans et plus et la femme 35 ans et plus [6]. Ces résultats sont illustrés à la Figure 1.

\section{AGE PATERNEL ET MALFORMATIONS}

Une publication de 1997 par une équipe française a mis en avant une majoration du risque de malformations chez les hommes de plus de 45 ans et a ainsi conseillé de fixer un âge limite de 45 ans pour les candidats donneurs de sperme [17]. En 2000, une publication originale montrait qu'entre 5 et $9 \%$ des cas de trisomie 21 (Down's syndrom) pourraient être d'origine paternelle [21].

Enfin une étude récente (2005) menée par une équipe danoise sur 71937 naissances montrait une augmentation de la prévalence de la trisomie 21 en fonction de l'âge paternel, passant de 1,2 chez les hommes âgés de 35-39 ans, à 1,3 pour ceux âgés de 40-44 ans, 1,7 pour ceux dans la tranche $45-49$ ans et enfin de 3,2 pour les hommes ayant plus de 50 ans [27].

\section{CONCLUSION}

L'analyse de la littérature montre donc une multiplication du nombre de publications internationales sur le sujet attestant ainsi de l'intérêt scientifique croissant d'une meilleure prise en compte de l'âge paternel sur la reproduction [5, 8]. Le Tableau 2 illustre les principales publications sur le sujet.

A la question " existe-t-il un effet de l'âge paternel sur la reproduction "), on peut donc raisonnablement répondre par l'affirmatif, altération des caractéristiques spermatiques, augmentation du risque d'échec de conception et augmentation du temps de conception, forte augmentation des risques de fausse-couche, majoration de certaines malformations congénitales. II reste bien sur à graduer cet effet de l'âge paternel sur la reproduction et si les risques semblent modérés entre 40 et 45 ans, ils sont probablement beaucoup plus importants lorsque le père est âgé de $\mathbf{4 5}$ ans et plus.

Parmi les nombreuses hypothèses évoquées, on retiendra d'une part des changements dans la 
Tableau 1 : Odds ratio ajustés $(95 \% \mathrm{Cl})$ du risque de conception après $F I V(n=1938)$.

(a) régression logistique (sans interaction âge maternel et paternel)

\begin{tabular}{lll}
\hline âge maternel (années) & \\
$<30$ & $(n=378)$ & 1,00 \\
$30-34$ & $(n=654)$ & $0,99(0,73-1,36)$ \\
$35-37$ & $(n=428)$ & $1,23(0,85-1,77)$ \\
$38-40$ & $(n=302)$ & $1,59(1,05-2,42)$ \\
$>40$ & $(n=176)$ & $2,21(1,28-3,80)$ \\
age paternel (années) & & \\
$<30$ & & \\
$30-34$ & $(n=276)$ & 1,00 \\
$35-39$ & $(n=597)$ & $1,52(1,08-2,14)$ \\
$\geq 40$ & $(n=585)$ & $1,32(0,92-1,89)$ \\
& $(n=480)$ & $1,70(1,14-2,52)$ \\
\hline
\end{tabular}

(b) régression logistique (avec l'interaction âge maternel et paternel)

\begin{tabular}{|c|c|c|c|c|c|}
\hline \multicolumn{2}{|c|}{ âge paternel (années) } & \multicolumn{2}{|c|}{ âge maternel (années) } & \multirow[b]{2}{*}{$38-40$} & \multirow[b]{2}{*}{$>40$} \\
\hline & $<30$ & $30-34$ & $35-37$ & & \\
\hline$<30$ & $\begin{array}{c}1.00 \\
\text { (référence) } \\
(n=145)\end{array}$ & $\begin{array}{c}0.79 \\
(0,42-1,51) \\
(n=63)\end{array}$ & $\begin{array}{c}1.62 \\
(0,57-4,57) \\
(n=27)\end{array}$ & $\begin{array}{c}1.29 \\
(0,48-3,43) \\
(n=27)\end{array}$ & $\begin{array}{c}0.49 \\
(0,16-1,50) \\
(n=14)\end{array}$ \\
\hline $30-34$ & $\begin{array}{c}1,44 \\
(0,84-2,46) \\
(n=152)\end{array}$ & $\begin{array}{c}1,34 \\
(0,84-2.13) \\
(n=283)\end{array}$ & $\begin{array}{c}1,49 \\
(0,78-2,85) \\
(n=86)\end{array}$ & $\begin{array}{c}1,47 \\
(0,65-3,33) \\
(n=45)\end{array}$ & $\begin{array}{c}5,34 \\
(1,22-23,42) \\
(n=31)\end{array}$ \\
\hline $35-39$ & $\begin{array}{c}0,78 \\
(0,40-1,50) \\
(n=59)\end{array}$ & $\begin{array}{c}1,24 \\
(0,76-2,02) \\
(n=205)\end{array}$ & $\begin{array}{c}1,33 \\
(0,80-2,22) \\
(n=180)\end{array}$ & $\begin{array}{c}3,05 \\
(1,44-6,48) \\
(n=93)\end{array}$ & $\begin{array}{c}2,16 \\
(0,89-5,20) \\
(n=48)\end{array}$ \\
\hline$\geq 40$ & $\begin{array}{c}1,25 \\
(0,43-3,62) \\
(n=22)\end{array}$ & $\begin{array}{c}1,36 \\
(0,75-2,46) \\
(n=103)\end{array}$ & $\begin{array}{c}2,00 \\
(1,10-3,61) \\
(n=135)\end{array}$ & $\begin{array}{c}2,03 \\
(1,12-3,68) \\
(n=137)\end{array}$ & $\begin{array}{c}5,74 \\
(2,16-15,23) \\
(n=83)\end{array}$ \\
\hline
\end{tabular}

(Données modifiées d'après référence [4]). 
Tableau 2 : Principales publications sur l'influence de l'âge paternel sur les issues reproductives.

\begin{tabular}{|c|c|c|c|}
\hline References & Populations étudiées & Issues reproductives & Effet de l'âge paternel? \\
\hline \multicolumn{4}{|l|}{ INFERTILITE } \\
\hline $\begin{array}{l}\text { La Rochebrochard et } \\
\text { Thonneau [7] }\end{array}$ & 3287 ayant essayé de concevoir & Infécondité (12 mois) & +++ \\
\hline Hassan et Killick [14] & 2112 grossesses & Délai de conception & +++ \\
\hline Dunson et al. [9] & $\begin{array}{l}782 \text { couples utilisant une méthode } \\
\text { naturelle de planification familiale }\end{array}$ & $\begin{array}{l}\text { Probabilité de concevoir selon } \\
\text { le jour du cycle }\end{array}$ & +++ \\
\hline Paulson et al. [20] & $\begin{array}{l}558 \text { cycles de FIV avec dons } \\
\text { d'ovules }\end{array}$ & Grossesses cliniques & - \\
\hline Watanabe et al. [26] & $\begin{array}{l}288 \text { cycles de FIV avec dons } \\
\text { d'ovules }\end{array}$ & Grossesses cliniques & + \\
\hline Ford et al. [11] & 8515 grossesses planifiées & Infécondité (6 à 12 mois) & ++ \\
\hline Gallardo et al. [12] & $\begin{array}{l}316 \text { cycles de FIV avec dons } \\
\text { d'ovules }\end{array}$ & Grossesses cliniques & - \\
\hline Joffe and Li [15] & $\begin{array}{l}2576 \text { hommes âgés de } 33 \text { ans et } \\
\text { ayant conçus }\end{array}$ & Délai de conception & - \\
\hline Ford et al. [10] & $\begin{array}{l}585 \text { couples ayant essayé de } \\
\text { concevoir }\end{array}$ & Infécondité (9 mois) & ++ \\
\hline Olsen [19] & 10886 grossesses & Infécondité (12 mois) & $-1+$ \\
\hline Nieschlag et al. [18] & 43 hommes ayant eu un enfant & Pénétration ovulaire & - \\
\hline \multicolumn{4}{|l|}{ FAUSSE-COUCHE } \\
\hline Astolfi et al. [2] & $\begin{array}{l}3,6 \text { millions de naissances et de } \\
\text { certificats de décès néo-nataux }\end{array}$ & Avortements tardifs & +++ \\
\hline Rychtarikova et al. [23] & $\begin{array}{l}598157 \text { naissances et certificats } \\
\text { de décès néo-nataux }\end{array}$ & Avortements tardifs & ++ \\
\hline Slama et al. [25] & $\begin{array}{l}1151 \text { femmes ayant eu } 2414 \\
\text { grossesses }\end{array}$ & Fausses-couches & +++ \\
\hline $\begin{array}{l}\text { de La Rochebrochard } \\
\text { et Thonneau [6] }\end{array}$ & 3174 grossesses planifiées & Fausses-couches & +++ \\
\hline Gourbin et Wunsch [13] & $\begin{array}{l}498122 \text { naissances et certificats } \\
\text { de décès néo-nataux }\end{array}$ & Avortements tardifs & + \\
\hline al-Ansary et Babay [1] & $\begin{array}{l}\text { patientes hospitalisées: } 226 \\
\text { fausses-couches } \\
\text { et } 226 \text { témoins }\end{array}$ & Fausses-couches & ++ \\
\hline Ford et al. [10] & 484 grossesses planifiées & $\begin{array}{l}\text { Fausses-couches (premier } \\
\text { trimestre) }\end{array}$ & ++ \\
\hline Selvin et Garfinkel [24] & $\begin{array}{l}1,5 \text { million de naissances et de } \\
\text { certificats de décès néo-nataux }\end{array}$ & Avortements tardifs & + \\
\hline Resseguie [22] & $\begin{array}{l}\text { naissances et certificats de } \\
\text { décès néo-nataux }\end{array}$ & Avortements tardifs & - \\
\hline
\end{tabular}

(Données modifiées d'après référence [5]). 
quantité et la qualité de la production spermatique et, d'autre part, une augmentation du risque de mutation dans les cellules germinales. Quant aux mécanismes, les auteurs évoquent une accumulation de lésions environnementales, une réduction de l'efficacité des réparations, une augmentation de l'instabilité génomique... [16, 21].

En conclusion et dans une période où on assiste à une augmentation des désirs d'enfant à des âges de plus en plus tardifs, ces résultats doivent certainement inciter le clinicien à avertir madame mais aussi monsieur d'une majoration des risques reproductifs liés à leur âge $[5,15]$.

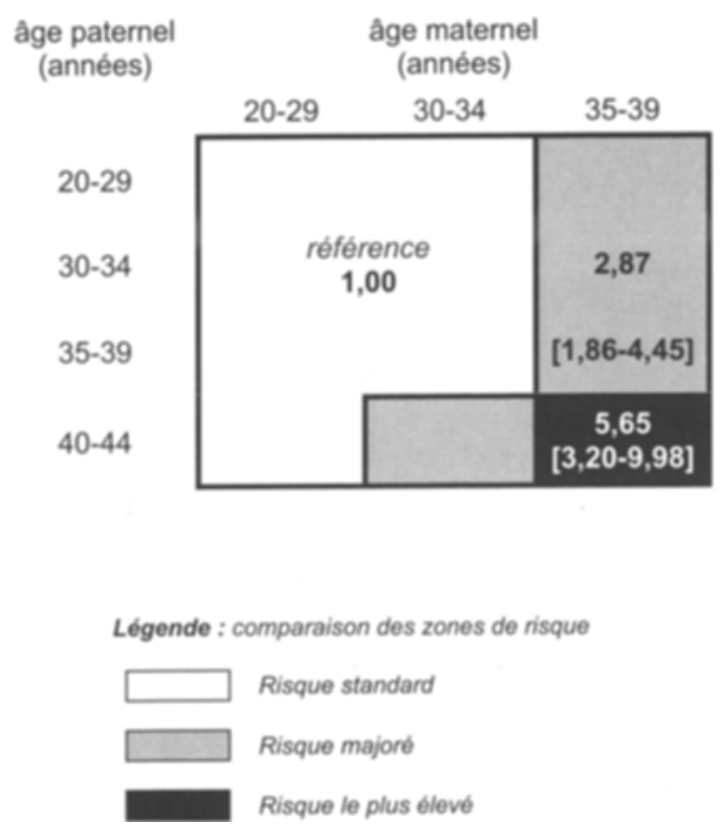

Figure 1 : Interaction entre l'âge maternel et paternel (2 414 grossesses). (Modifiée d'après [6]).

\section{REFERENCES}

1. AL-ANSARY L.A., BABAY Z.A. : Risk factors for spontaneous abortion: a preliminary study on Saudi women. J. R. Soc. Health, 1994, 114: 188-193.

2. ASTOLFI P., DE PASQUALE A., ZONTAL.A. : Late paternity and stillbirth risk. Hum. Reprod., 2004, 19: 2497-2501.

3. BONDE J.P., ERNST E., JENSEN T.K., HJOLLUND N.H., KOLSTAD H., HENRIKSEN T.B. et al. : Relation between semen quality and fertility : a population-based study of 430 first-pregnancy planners. Lancet, 1998, 352 : 1172-1177.

4. DE LA ROCHEBROCHARD E., DE MOUZON J., THEPOT F., THONNEAU P. and the French National IVF Registry (FIVNAT) Association : Fathers over 40 and increased failure to conceive : the lessons of in vitro fertilization in France. Fertil. Steril., 2006, $85:, 1424$.
5. DE LA ROCHEBROCHARD E., MCELREAVEY K., THONNEAU P. : Paternal age over 40 years : the 'amber light' in the reproductive life of men ? J. Androl., 2003, 24 : 459465.

6. DE LAROCHEBROCHARD E., THONNEAU P. : Paternal age and maternal age are risk factors for miscarriage ; results of a multicentre European study. Hum. Reprod., 2002, 17: 16491656.

7. DE LAROCHEBROCHARD E., THONNEAU P. : Paternal age over 40 years : an important risk factor for infertility. Am. J. Obstet. Gynecol., 2003, $189: 901-905$.

8. DE LA ROCHEBROCHARD E, THONNEAU P. : Paternal age : are the risks of infecundity and miscarriage higher when the man is aged 40 years or over ? Rev. Epidemiol Santé Publique, 2005, 53 : 2S47-2S55

9. DUNSON D.B., COLOMBO B., BAIRD D.D. : Changes with age in the level and duration of fertility in the menstrual cycle. Hum. Reprod., 2002, 17 : 1399-1403.

10. FORD J.H., MACCORMAC L., HILLER J., PALS (pregnancy and lifestyle study) : Association between occupational and environmental exposure to chemicals and reproductive outcomes. Mutat. Res., 1994, 313 : 153-164.

11. FORD W.C., NORTH K., TAYLOR H., FARROW A., HULL M.G., GOLDING J. : Increasing paternal age is associated with delayed conception in a large population of fertile couples : evidence for declining fecundity in older men. The ASPALC Study Team (Avon longitudinal Study of Pregnancy and Childhood). Hum. Reprod., 2000, $15:$ 1703-1708.

12. GALLARDO E., SIMON C., LEVY M., GUANES P.P., REMOHI J., PELLICER A. : Effect of age on sperm fertility potential : oocyte donation as a model. Fertil. Steril., 1996, $66: 260-264$.

13. GOURBIN C., WUNSCH G. : Paternal age and infant mortality. Genus, 1999, LV : 61-72.

14. HASSAN M.A., KILLICK S.R. : Effect of male age on fertility: evidence for the decline in male fertility with increasing age. Fertil. Steril., 2003, $79: 1520-1527$.

15. JOFFE M., LI Z. : Male and female factors in fertility. Am. J. Epidemiol., 1994, $140:$ 921-929.

16. KIDD S.A., ESKENAZI B., WYROBEK A.J. : Effects of male age on semen quality and fertility : a review of the literature. Fertil. Steril., 2001, $75:$ 2237-2248.

17. LANSAC J., THEPOT F., MAYAUX M.J., CZYGLICK F., WACK T., SELVA J., JALBERT P. : Pregnancy outcome after artificial insemination or IVF with frozen semen donor : a collaborative study of the French CECOS Federation on 21, 597 pregnancies. Eur. J. Obstet. Gynecol. Reprod. Biol., 1997, 74: 223-228.

18. NIESCHLAG E., LAMMERS U., FREISCHEM C.W., LANGER K., WICKINGS E.J. : Reproductive functions in young fathers and grandfathers. J. Clin. Endocrinol. Metab., 1982, 55 : 676681.

19. OLSEN J. : Subfecundity according to the age of the mother and the father. Dan. Med. Bull., 1990, $37: 2812$.

20. PAULSON R.J., MILLIGAN R.C., SOKOL R.Z. : The lack of influence of age on male fertility. Am. J. Obstet. Gynecol., 2001, $184: 818-822$. 
21. PETERSEN M.B., MIKKELSEN M. : Nondisjunction in trisomy 21 : origin and mechanisms. Cytogenet. Cell. Genet., 2000, 91 : 199-203.

22. RESSEGUIE L.J. Paternal age, stillbirths and mutation. Ann. Hum. Genet., 1976, $40: 213-219$.

23. RYCHTARIKOVA J., GOURBIN C., WUNSCH G. : Paternal age and child death : the stillbirth case. Eur. J. Popul., 2004, $20: 23-33$.

24. SELVIN S., GARFINKEL J. : Paternal age, maternal age and birth order and the risk of a fetal loss. Hum. Biol., 1976, 48 : 223-230.

25. SLAMA R., WERWATZA., BOUTOU O., DUCOT B., SPIRAL A., HARDLE W. : Does male age affect the risk of spontaneous abortion ? An approach using semiparametric regression. Am. J. Epidemiol., 2003, $157:$ 815-824.

26. WATANABE Y., CORNET D., MERVIEL P., MANDELBAUM J., ANTOINE J.M., UZAN S. : Influence of husband's age on outcome of a shared oocyte donation program. Fertil. Steril., 2000, 74 : S78-S79.

27. ZHU J.L., MADSEN K.M., VESTERGAARD M., OLSEN A.V., BASSO O., OLSEN J. : Paternal age and congenital malformations. Hum. Reprod., 2005, $20: 3173-3177$.

Manuscrit reçu : janvier 2007 ; accepté mars 2007.

\section{ABSTRACT}

Does advanced paternal age constitute a reproductive risk factor?

Patrick THONNEAU, Elise de LA ROCHEBROCHARD

If it is now clearly established that advanced maternal age can have a deleterious impact on reproductive issues, and an increasing number of recent publications also indicate an increased risk associated with advanced paternal age. Based on a review of the literature, the authors analyse the consequences of advanced paternal age on various reproductive parameters.

Key words : fertility, male fertility, reproduction, paternal age, epidemiology 